\title{
Optimization of MPPT Step Size in Stand-alone Solar Pumping Systems
}

\author{
Songbai Zhang, Zheng Xu, Youchun Li, and Yixin Ni, Senior Member, IEEE
}

\begin{abstract}
In a stand-alone solar pumping system with a pump driven by an induction motor, the step size in maximum power point tracking (MPPT) methods greatly affects the dynamics and stability of the system. In this paper, we analyze some phenomena appearing in the contrastive experiments which have been carried out for about one year, and give a solution to determine the step size based on system parameters and controller capabilities. The contrastive experiments show that the system performance can be obviously improved by the optimized step size together with an adequate smoothing capacitor.
\end{abstract}

Index Terms--inverter, maximum power point tracking, photovoltaic array, solar pumping system

\section{INTRODUCTION}

A $\mathrm{s}$ the conventional fossil energy resources are rapidly dwindling, solar energy offers a very promising alternative. Photovoltaic (PV) arrays produce electric power directly from sunlight with no fossil-fuel consumption, no noise, and posing no health and environmental hazards. In all of the PV applications, solar pumping systems applied in remote areas have received considerable attention [1]. In a solar pumping system with a water pump driven by an induction motor, DC voltage of PV array is converted into three-phase AC voltage by a DC-AC inverter. To improve the efficiency of photovoltaic systems, the maximum power point tracking (MPPT) function is necessary. Several methods have been proposed, such as Constant Voltage (CV) method [2], Perturb and Observe (PAO) method [3]-[4] and Incremental Conductance Technique (ICT) [3], [5], and they are valid in many applications. However, when applying these methods to a stand-alone solar pumping system, the performance is not satisfactory. In another paper [6], the authors have discussed the problem and proposed a novel hybrid MPPT control method that can offer good dynamics as well as stability.

The project is financed by Shenzhen Bureau of Science Technology and Information.

Songbai Zhang is with the department of Electrical Engineering, Graduate School of Tsinghua University at Shenzhen, P.R.CHINA (e-mail: zhangsb03@mails.tsinghua.edu.cn).

Zheng Xu is with the National Key Laboratory of Power Systems at Shenzhen, P.R. China (e-mail: xuz@mail.sz.tsinghua.edu.cn).

Youchun $\mathrm{Li}$ is with the Shenzhen Highest Power Technology Co. Ltd (e-mail: leeych@gmail.com).

Yixin $\mathrm{Ni}$ is with Electric and Electronic Engineer Department, the University of Hong Kong (e-mail: yxni@eee.hku.hk).
During the course of the contrastive experiments, we find that the step size in the MPPT methods affect the performance of the system. In order to realize an accurate MPPT, the step size is desired to be as small as possible. Generally, it is easy for an inverter to realize an output frequency precision of $0.01 \mathrm{~Hz}$. On the other hand, a small step size will slow down the tracking speed and degrade the dynamics. The contrastive experiments over about one year show that the output power sometimes cannot track the maximum power point (MPP) well when using a small step size.

We investigated the influence of step size with different MPPT methods, in different seasons and under different weather conditions. We find that the acceptable minimum step size mainly depends on the accuracy of sensors and A/D converters of the controller. A method of step size optimization that takes system parameters and controller capabilities into account is proposed in this paper. Its effect on improvement of MPPT has been verified experimentally.

\section{System Configuration And Control Method}

The research of this paper is based on the project of standalone solar pumping systems for irrigation of the highway shelterbelt in Xinjiang Uygur Autonomous Region, China. The highway is about 500 kilometers long, crossing Taklamakan Desert from north to south where no electric grid is available. For the purpose of low cost and easy maintenance, we choose a simple system consisting of three parts: a PV array, an application specific inverter with an MPPT controller, and a pump with a three-phase induction motor. No backup battery is used in the system. Fig.1 shows a simplified structure of solar pumping systems.

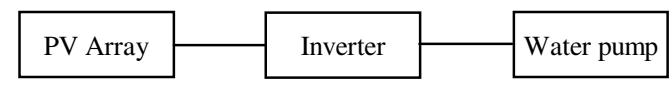

Fig. 1. Structure of stand-alone solar pumping systems

\section{A. Output characteristics of PV array}

The output power of a PV array mainly depends on insolation condition and ambient temperature [7]. The voltageampere characteristic of a PV array is expressed by the following equation.

$$
I=I_{p v}-I_{s c}\left(\exp \left(\frac{q\left(V+I R_{s}\right)}{n k T}\right)-1\right)-\frac{V+I R_{s}}{R_{s h}}
$$

where $I$ : output current;

$$
V: \text { output voltage; }
$$


$I_{s c}:$ cell reverse saturation current;

$I_{p v}$ : light-generated current;

$q$ : charge of an electron;

$K$ : Boltsman's constant;

$T$ : cell temperature in $\mathrm{K}$;

$n$ : factor of $\mathrm{p}-\mathrm{n}$ junction;

$R_{s h}:$ shunt resistance

$R_{s}$ : serial resistance

The corresponding I-V and P-V curves are shown in Fig.2. Corresponding to a certain insolation, the PV array has only one MPP which divides the output I-V curve into two parts: the left part is defined as the current source region in which the output current approximately is constant, and the right part is the voltage source region in which the output voltage does not change much.

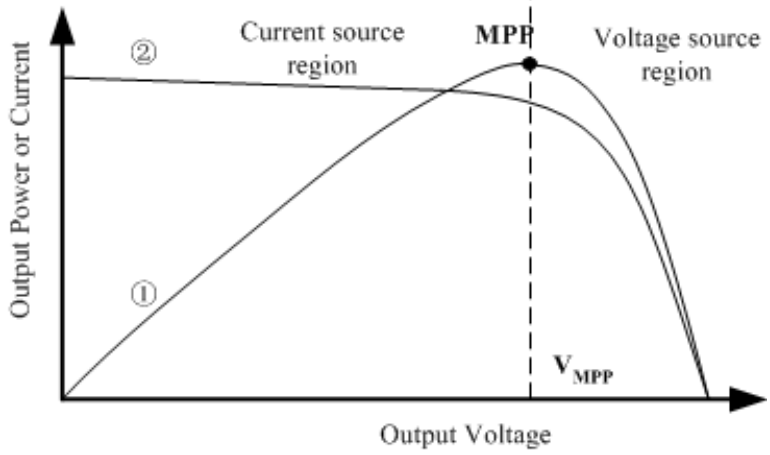

Fig. 2. P-V (I-V) characteristic of a PV array

In a stand-alone solar pumping system without backup batteries, the operating point of the PV array can be controlled by the output frequency regulation of the inverter. The MPP is tracked by means of increasing frequency in the voltage source region and decreasing frequency in the current source region. One point should be highlighted that the system with a load of an induction motor is unstable in the current source region. Because in that region, the output voltage of the PV array will rapidly fall, and the system will enter low voltage protection mode if the frequency is not decreased in time. So a good dynamic performance of the controller is desired.

\section{B. Control methods of MPPT}

In previous work, we compared the performances of $\mathrm{CV}$, PAO and ICT method in solar pumping systems, and proposed a novel hybrid control method that offers good dynamics as well as stability [6].

The hybrid method combines $\mathrm{CV}$ method with a multicriterion (MC) method. The principle of CV method is based on the assumption that the MPP voltage keeps a constant value The output frequency is determined by comparing the output voltage of $\mathrm{PV}$ array with its reference $V_{\text {ref }}$.

$$
f(n)= \begin{cases}f(n-1)+\Delta f & V(n)>V_{r e f} \\ f(n-1) & V(n)=V_{r e f} \\ f(n-1)-\Delta f & V(n)<V_{r e f}\end{cases}
$$

$\Delta f$ is the step size of the output frequency and $\Delta f>0$.

In $\mathrm{MC}$ method we proposed in [6], $\Delta P$ and $\Delta V$ are used to track the MPP, and $\Delta I$ is used to judge the change of insolation to improve the system dynamics. The detail control rule is summarized in Table I.

The system with the hybrid method is basically controlled in $\mathrm{CV}$ method while its reference voltage is periodically updated by MC method as shown in Fig.3. The two methods are active by turns. The period of the CV method (for example 5 seconds each time) is much longer than that of MC method (less than 1 second). The system possesses both the fine tracking precision of the MC method and the good stability of $\mathrm{CV}$ method.

TABLE I

LOGIC OF THE MULTI-CRITERION METHOD

\begin{tabular}{ccccc}
\hline & Criterion & & Frequency & System State \\
$\Delta P$ & $\Delta V$ & $\Delta I$ & $f(\mathrm{n})$ & \\
\hline$<0$ & $<0$ & $<0$ & $f(n-1)-\Delta f$ & insolation decreasing \\
$<0$ & $<0$ & $\geqslant 0$ & $f(n-1)-\Delta f$ & current source \\
$<0$ & $\geqslant 0$ & $<0$ & $f(n-1)+\Delta f$ & voltage source \\
$>0$ & $<0$ & $\geqslant 0$ & $f(n-1)+\Delta f$ & voltage source \\
$>0$ & $\geqslant 0$ & $<0$ & $f(n-1)-\Delta f$ & current source \\
$>0$ & $>0$ & $>0$ & $f(n-1)+\Delta f$ & insolation increasing \\
$=0$ & - & - & $f(n-1)$ & MPP \\
\hline
\end{tabular}

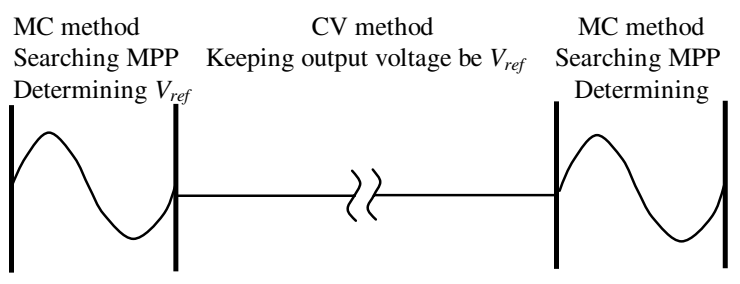

Fig. 3. The hybrid MPPT method

The step size $\Delta f$ during each control period is a very important element that affects the system dynamics and stability. A large step size leads to fast dynamic response but a large vibration around the MPP. A small step size leads to a good stability, but it takes a long time to approach the MPP. In order to combine the merits of large and small step size, we tried to select the step size based on the voltage of the PV array.

$$
\Delta f=\left\{\begin{array}{lc}
k\left(V_{1}-V\right)+\Delta f_{\min } & V<V_{1} \\
\Delta f_{\min } & V_{1} \leq V \leq V_{2} \\
k\left(V-V_{2}\right)+\Delta f_{\min } & V>V_{2}
\end{array}\right.
$$

where, $V_{1}<V_{r e f}<V_{2}, V_{r e f}$ is the MPP voltage.

This method is equal to a proportional control with a dead band $\left(V_{1}, V_{2}\right)$ that has been properly chosen. In order to reduce the vibration around the MPP, the minimum step size is selected when the voltage is within the dead band. Outside the dead band, the farther the system is away from the MPP, the larger the step size is selected. So the convergence time to the MPP is shortened.

\section{Experimental system and results}

In order to compare the control characteristics of different methods, two identical experimental systems are implemented in Shenzhen as shown in Fig.4.

The parameters of the PV array in each system are as follows, and the test results of output characteristics of the PV arrays are 
shown in Fig.5.

peak power $=2200 \mathrm{~W}$

MPP voltage $=350 \mathrm{~V}$

MPP current $=6.28 \mathrm{~A}$

The parameters of the submergible pumps are as follows:

rated voltage $=220 \mathrm{~V}_{\text {rms }}$

rated power $=1500 \mathrm{~W}$

flux $/$ head $=14\left(\mathrm{~m}^{3} / \mathrm{h}\right) / 20 \mathrm{~m}$

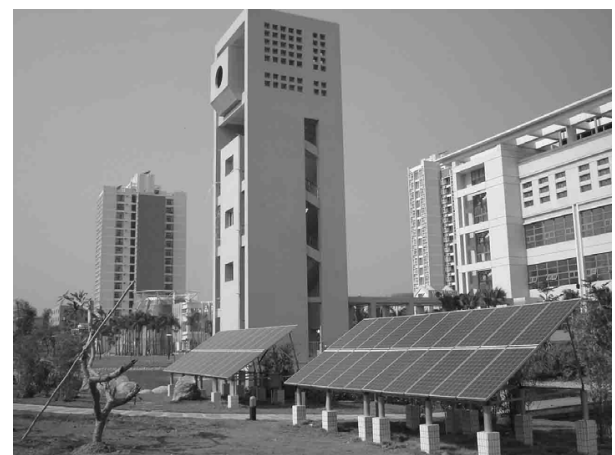

Fig. 4. Stand-alone solar pumping experimental systems in Shenzhen

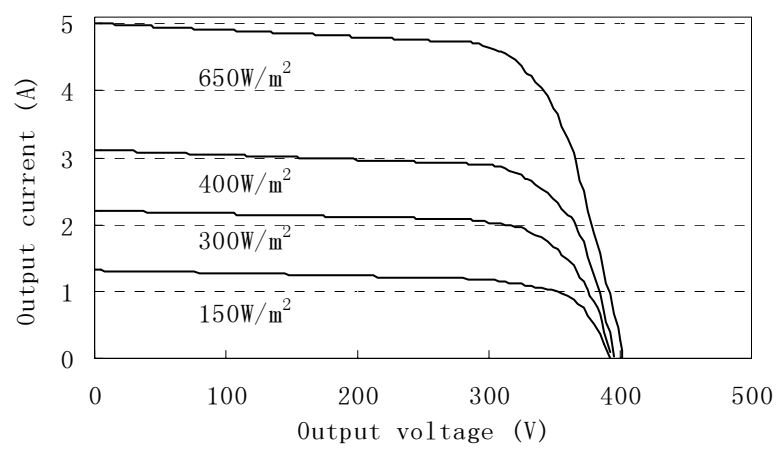

(a) I-V curves under different insolations

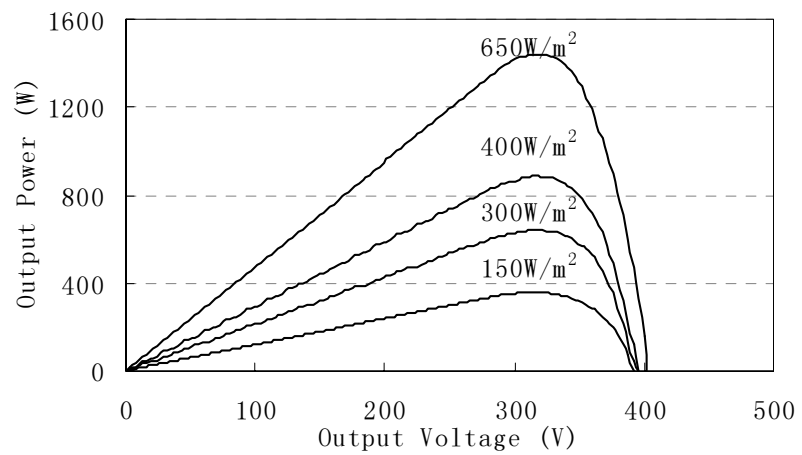

(b) P-V curves under different insolations

Fig. 5. Test results of output characteristics of PV array

The application specific inverter is designed based on a 16 bit fixed-point microprocessor, and several MPPT control methods are valid for experiment. In order to perform experiments for different water-heads, the outlets are set at four heights $(6,11,16$ and $19 \mathrm{~m})$.

The experiments are carried out from early in the morning to late in the afternoon. During the experiments, the output voltage and current of PV arrays are recorded every 5 seconds. The results shown below are under the condition of using the hybrid method, selecting 11 meters high outlets and limiting the output frequency range from 20 to $50 \mathrm{~Hz}$.

Fig. 6 - Fig. 8 show the output power and output voltage of the PV array under three typical weather conditions.

Fig.6 is the result obtained in a cloudless day. Under the condition of slow insolation change, the system can realize MPPT with very small disturbance. The output power reaches a peak value of $1470 \mathrm{~W}$, and the curve is completely in accordance with the level of insolation. The output voltage slightly varies in a range from 325 to $350 \mathrm{~V}$.
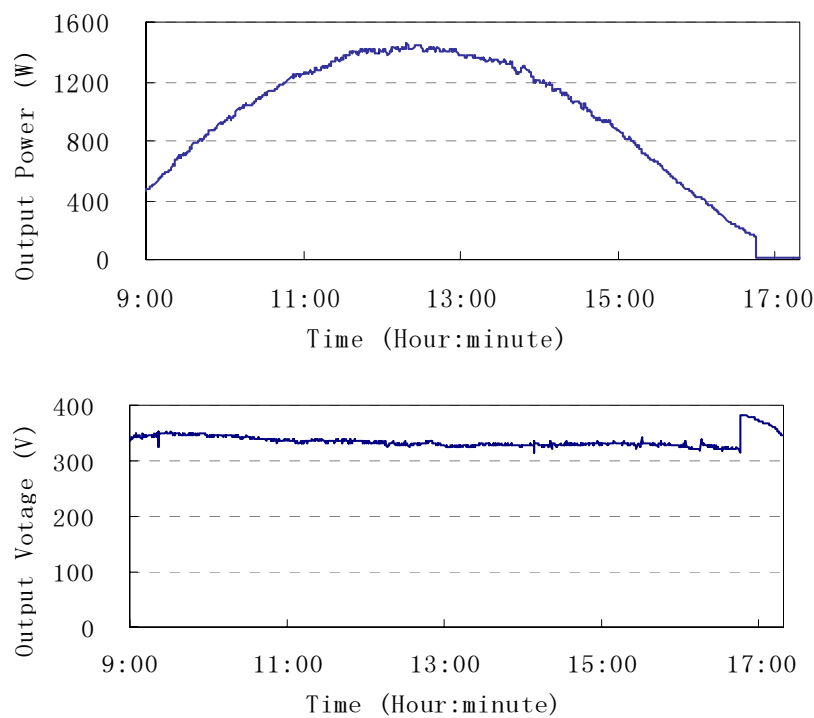

Fig. 6. Experimental results in a cloudless day (10 Jan. 2005)

Fig.7 is the result obtained in a day with cloud drift. The system can withstand the bad condition of rapid insolation variation and tracks MPP very well. The output power rapidly sways between $500 \mathrm{~W}$ to $1600 \mathrm{~W}$, but the output voltage stays in 320 to $350 \mathrm{~V}$.
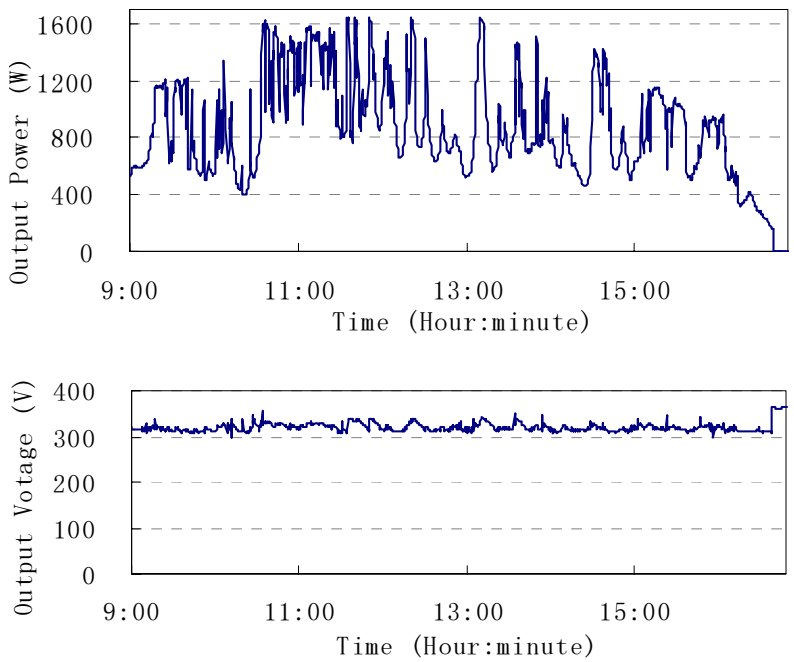

Fig. 7. Experimental results in a day with cloud drift (25 May 2005)

Fig. 8 is the result obtained in a cloudy day. The output power is less than $600 \mathrm{~W}$, and the output voltage is also within the range of 320 to $350 \mathrm{~V}$. 

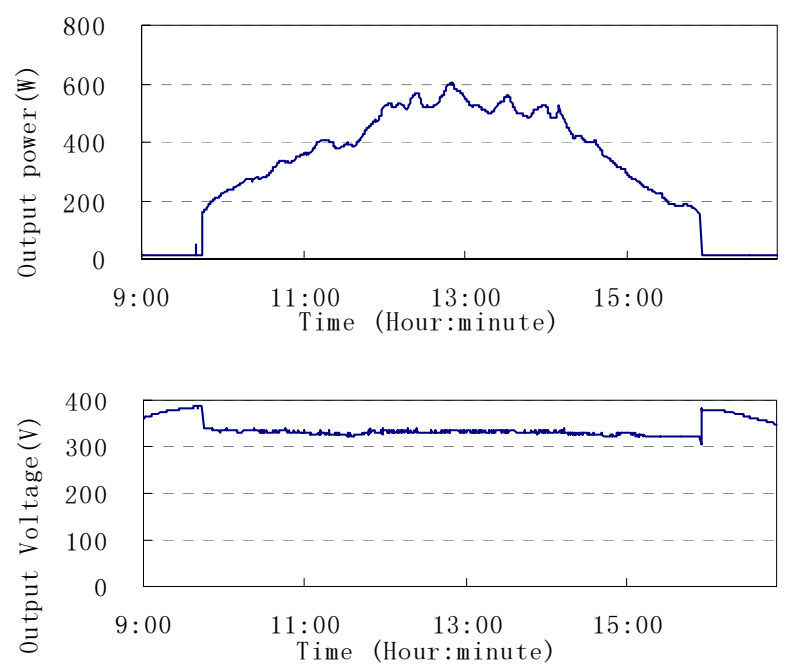

Fig. 8. Experimental results in a cloudy day (23 Dec. 2004)

\section{OPTIMIZATION OF STEP SIZE}

Theoretically a system with the above mentioned hybrid method could be expected always having a very good performance as shown in Fig.6 - Fig.8. But in both of the experimental systems, we sometimes met with unexpected operating states.

\section{A. Abnormal phenomena and analysis}

From the experimental results obtained for about one year, we find some abnormal phenomena. The abnormal phenomenon is that the one of the experimental systems sometimes works with output power smaller than the MPP for period of time.

1. The two contrastive systems sometimes operated at different points even with identical settings and conditions, as shown in Fig.9. The abnormal system obviously was away from the maximum power from 11:00 to 14:00. During this time, the abnormal system operated with output power smaller than that of the normal system by $200 \mathrm{~W}-400 \mathrm{~W}$.

2. Both of the systems are possible to fall into abnormal operation, with no regard to weather conditions and MPPT methods (PAO, ICT, MC and hybrid method). Table II shows the number of days that the system had a bad tracking performance in each month from January 2005 to August 2005. During these days, the instant lost power reached a peak value of $50 \%$, and the average lost energy was about $15 \%$.

3 . The system became normal when we interrupted its operation and restarted it again. So the MPPT method itself is not the cause.

Through further study, we finally arrived at a simple conclusion: the setting value of the minimum step size $\Delta f_{\min }$ is too small. Since the system usually stays at a wrong working point for quite a long time, it is reasonable to doubt whether the system misjudges MPP or not. In MPPT process, a judgment is made based on the variations of output voltage, current or power $(\Delta V, \Delta I$ or $\Delta P)$ caused by $\Delta f$ during last control period. The criterions of an MPP in different methods are as follows.

PAO method: $\Delta P=0$

ICT method: $I / V+\Delta I / \Delta V=0$

MC method: $\quad \Delta P=0$, where, $\Delta P$ is proportional to $\Delta V$ and $\Delta I$.
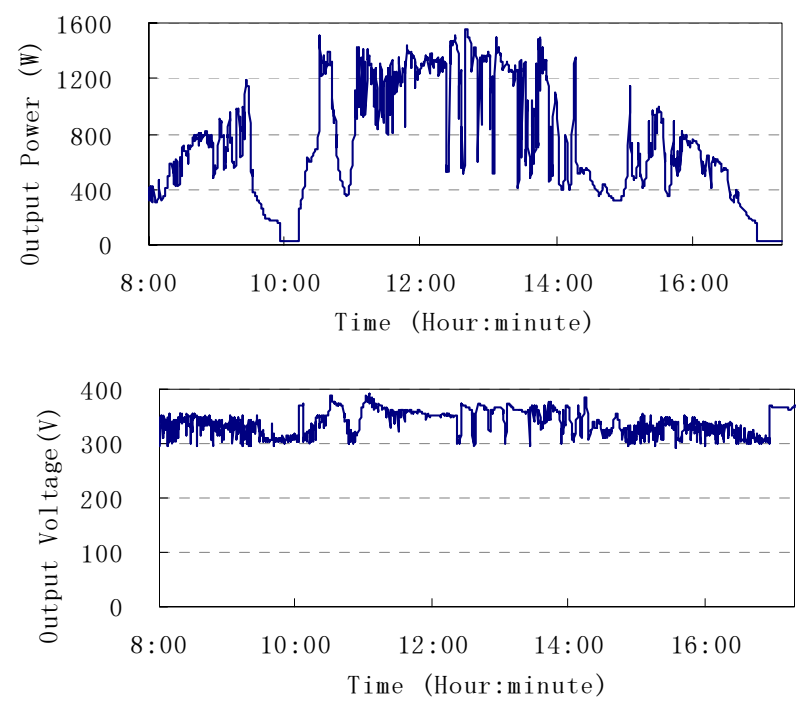

(a) Normal system
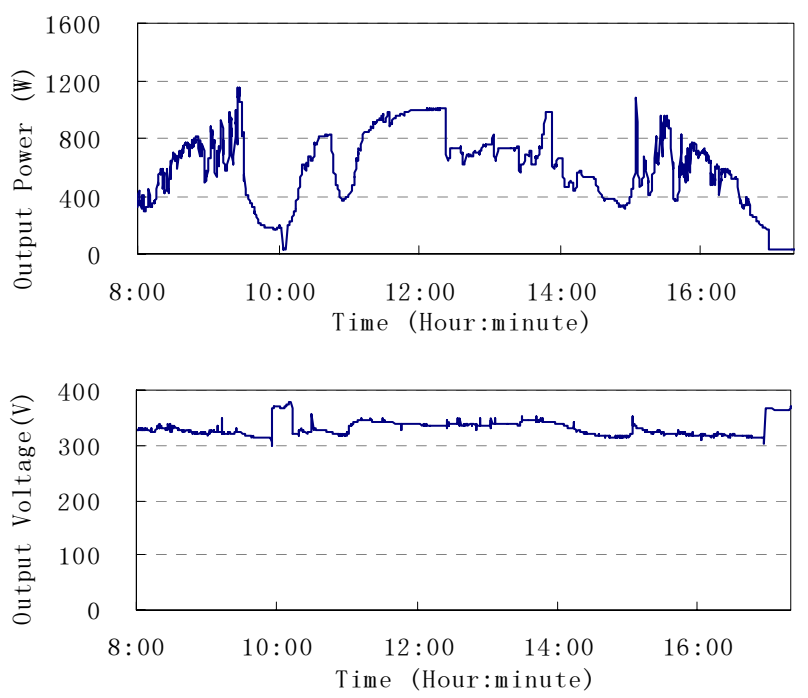

(b) Abnormal system

Fig. 9. Abnormal phenomenon (22 Apr. 2005)

TABLE II

DAYS OF ABNORMAL OPERATION IN EACH MONTH IN 2005

\begin{tabular}{|c|c|c|c|c|c|c|c|c|}
\hline system & Jan & Feb & Mar & Apr & May & Jun & Jul & Aug \\
\hline $1 \#$ & 4 & 2 & 5 & 6 & 6 & 4 & 6 & 3 \\
\hline $2 \#$ & 3 & 4 & 6 & 3 & 7 & 4 & 6 & 4 \\
\hline Total & 7 & 6 & 11 & 9 & 13 & 8 & 12 & 7 \\
\hline
\end{tabular}

According to (3), we use a large variable step size when the output voltage is far from the MPP to keep a high tracking speed. Otherwise, we use a small constant step size $\Delta f_{\min }$ to reduce vibrations around the MPP. The problem is how large $\Delta V, \Delta I$ and $\Delta P$ are for a given $\Delta f_{\min }$. If $\Delta V, \Delta I$ and $\Delta P$ are too small, the controller will be unable to resolve them. In order to make vibrations as small as possible, we have selected a very small value $(0.01 \mathrm{~Hz})$ as $\Delta f_{\min }$ in the above-mentioned experiments.

On the other hand, the sensitivity of a controller depends on the gain of sensors and the precision of $\mathrm{A} / \mathrm{D}$ converters. In our experimental systems, with a 10-bit A/D converter in the 
microprocessor unit (MPU), the resolutions of output voltage and current are $0.5 \mathrm{~V} / \mathrm{bit}$ and $0.033 \mathrm{~A} / \mathrm{bit}$ respectively, that means if $\Delta V<0.5 \mathrm{~V}$ and $\Delta I<0.033 \mathrm{~A}$, the controller will treat the present working point as the MPP even it is not. To grasp how output voltage and current vary with the output frequency, we made an actual measurement with the experimental systems. The V-f and I-f curves in Fig.10 show that in the MPPT range of frequency (from $20 \mathrm{~Hz}$ to $50 \mathrm{~Hz}$ ), the output voltage and current have average slopes of $-1.72 \mathrm{~V} / \mathrm{Hz}$ and $0.1468 \mathrm{~A} / \mathrm{Hz}$, maximum slopes of $-6.6 \mathrm{~V} / \mathrm{Hz}$ and $0.3594 \mathrm{~A} / \mathrm{Hz}$, respectively. Based on the average slopes, one-bit values of the output voltage and current correspond to $0.29 \mathrm{~Hz}$ and $0.23 \mathrm{~Hz}$. Therefore, if $\Delta f_{\min }$ is smaller than $0.23 \mathrm{~Hz}$, the corresponding $\Delta V$ and $\Delta I$ will be too small to be detected by the controller. Even with the maximum slopes, $\Delta f_{\min }$ should be larger than $0.08 \mathrm{~Hz}$. Therefore, it is easy to understand why the system failed to track the MPP with a quite high probability when $\Delta f_{\min }=0.01 \mathrm{~Hz}$.
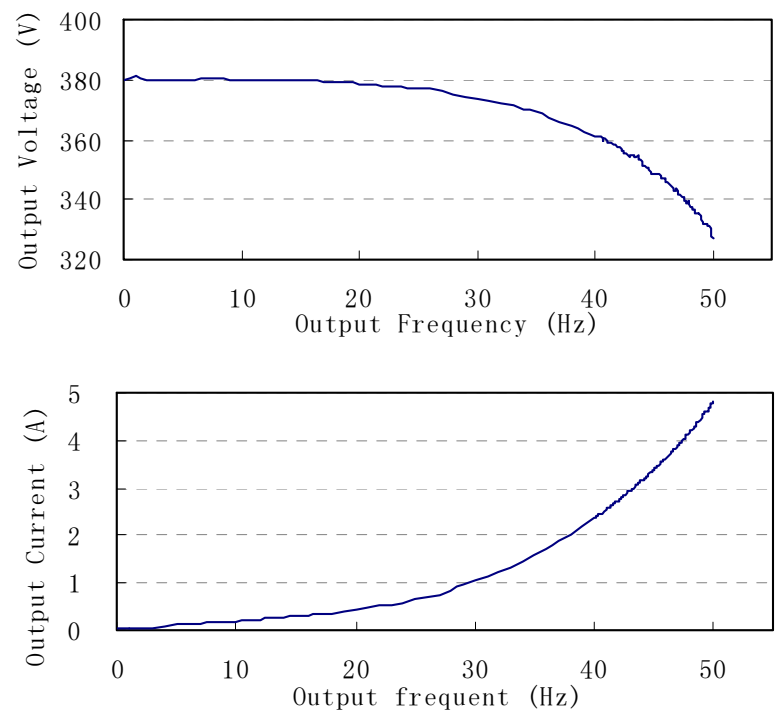

Fig. 10. $V-f$ and $I-f$ curves of the experimental systems

\section{B. Optimization of minimum step size}

According to the above analysis, contrastive experiments on different $\Delta f_{\min }$ have been carried out. As shown in Fig.11, the system with $\Delta f_{\min }=0.01 \mathrm{~Hz}$ becomes abnormal since 12 o'clock and lasts for about 4 hours, while another system with $\Delta f_{\min }=$ $0.5 \mathrm{~Hz}$ tracks the MPP very well but its vibration around the MPP is obvious.

In general, the MPPT step size for a stand-alone solar pumping system can be determined by two basic rules. Firstly, a large variable step size is adopted when the present working point is far from MPP, a small constant step size when close to MPP. Secondly, $\Delta f_{\min }$ is determined on the basis of system parameters and MPU capabilities. Experientially, for a system with well-matched PV array and load, we can calculate the optimal value of $\Delta f_{\min }$ by following equations.

$$
\Delta f_{\min } \approx \max \left[\frac{2^{-N} f_{\text {rate }} V_{a d}}{K_{I} I_{p}}, \frac{2^{-N} f_{\text {rate }} V_{a d}}{K_{V}\left(V_{o}-V_{p}\right)}\right]
$$

where $V_{o}: \quad$ open circuit voltage of PV array

$V_{p}: \quad$ voltage of $\mathrm{PV}$ array at peak power point

$I_{p}: \quad$ current of PV array at peak power point
$F_{\text {rate }}:$ rated frequency of pump

$K_{V}:$ gain of voltage sensor

$K_{I}$ : gain of current sensor

$V_{a d}$ : reference voltage of A/D converter

$N$ : bit number of $\mathrm{A} / \mathrm{D}$ converter

In the case of our experimental systems,

$V_{o}=425 \mathrm{~V}, \quad V_{p}=350 \mathrm{~V}, \quad I_{p}=6.28 \mathrm{~A}$,

$f_{\text {rate }}=50 \mathrm{~Hz}, \quad K_{V}=0.01, \quad K_{I}=0.15$

$V_{a d}=5 \mathrm{~V}, \quad N=10$

the step size calculated by Eq. 4 is $0.33 \mathrm{~Hz}$. In the experiments started from November $5 \mathrm{th}, 2005$, the system with $\Delta f_{\min }=0.3 \mathrm{~Hz}$ have been working very well while another system with $\Delta f_{\text {min }}=0.01 \mathrm{~Hz}$ failed to track the MPP 4 times during the same period of time.
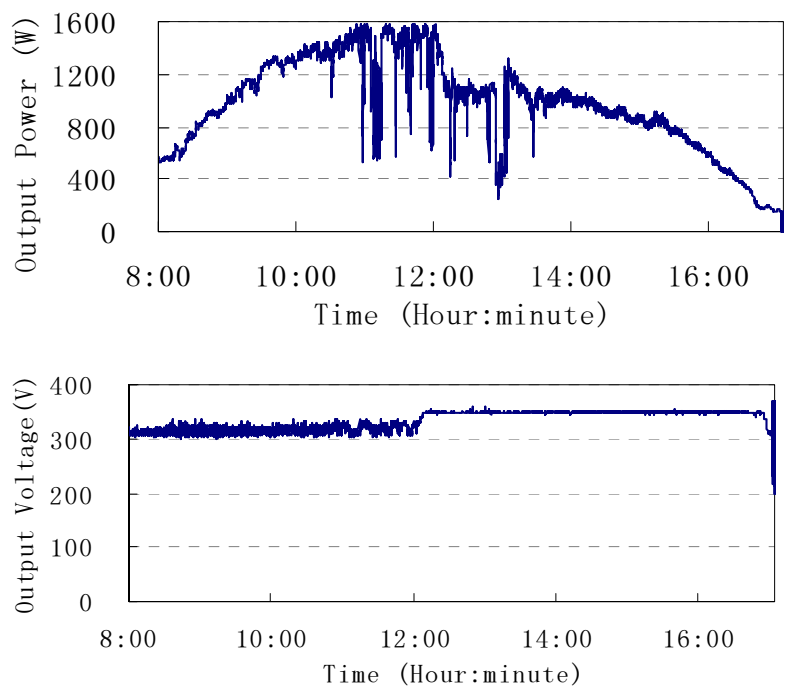

(a) The system with $\Delta f_{\min }=0.01 \mathrm{~Hz}$
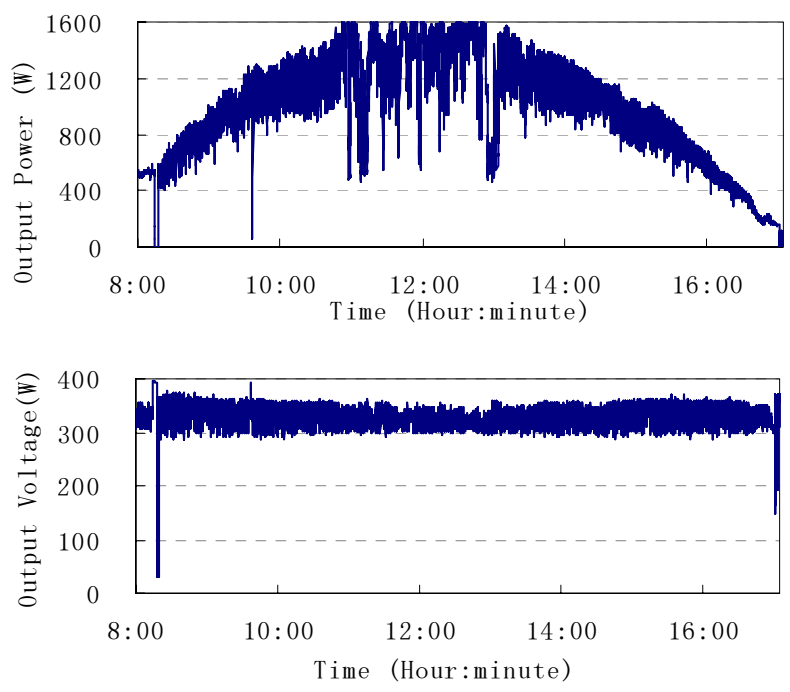

(b) The system with $\Delta f_{\text {MIN }}=0.5 \mathrm{~Hz}$

Fig. 11. Experimental results with different step sizes (3 Nov. 2005)

\section{Effect of smoothing capacitor}

Principally, it is not necessary to install a smoothing capacitor into the inverter of a solar pumping system because the PV array directly supplies DC voltage. However, we try to improve the system performance by adding a capacitor parallel 
to the PV array. A few experiments with different capacitors $(560 \mu \mathrm{F}, 2760 \mu \mathrm{F}$ and $6160 \mu \mathrm{F})$ were made. It is clear from the results that a large capacitor has two nice effects on the system. First, it can gain time for the controller to prevent the system from collapsing when the insolation rapidly falls. Secondly, it can reduce the vibration around the MPP caused by a large step size. From the curves of output power and voltage $\left(\Delta f_{\min }=0.3 \mathrm{~Hz}\right)$ in Fig.12, we can see the vibration of the system with a large capacitor is much smaller. So we suggest installing a smoothing capacitor in the system.
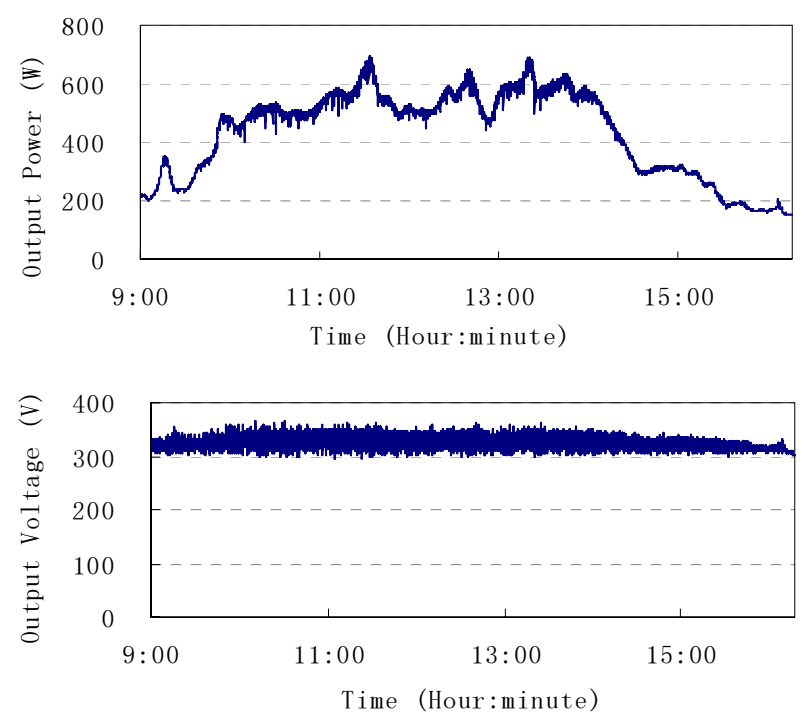

(a) The system with $\mathrm{C}=560 \mu \mathrm{F}, \Delta f_{\min }=0.3 \mathrm{~Hz}$
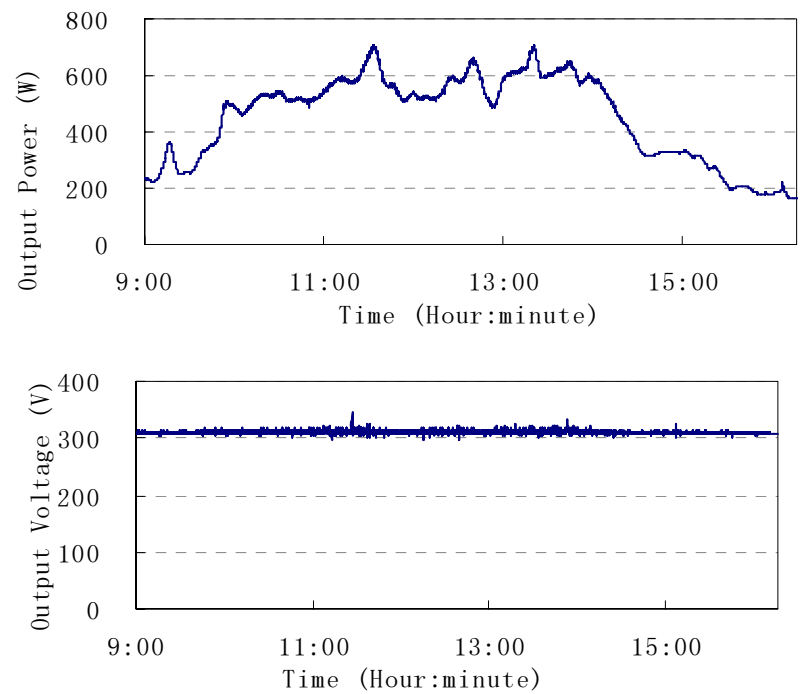

(b) The system with $\mathrm{C}=2760 \mu \mathrm{F}, \Delta f_{\min }=0.3 \mathrm{~Hz}$

Fig. 12. Experimental results with different capacitors (31 Oct. 2005)

\section{CONCLUSION}

In a stand-alone solar pumping system, the step size of MPPT method is an important factor that influences control characteristics. From the experimental results over a long period of time, we can draw several useful conclusions.

1. Adopting a large variable step size when the present working point is far from MPP, and the minimum step size when close to MPP, is an effective way to make a good dynamics as well as stability.

2. If the minimum step size is too small, the system may fail to track MPP.

3. The optimal value of the minimum step size depends on system parameters and controller capabilities, and can be calculated by the approximate formula we proposed in this paper.

4. A smoothing capacitor parallel to the PV array is useful to prevention against collapse, and reduction of vibration.

\section{REFERENCES}

[1] Kirk Stokes, and John Bigger, "Reliability, Cost, and Performance of PVpowered Water Pumping Systems: A Survey For Electric Utilities" IEEE Transactions on Energy Conversion, Vol. 8, No. 3, September 1993, pp.506-512.

[2] Arrouf M., and Goedel C. "Photovoltaic pumping system for induction machine with hysteresis array current control," IEEE AFRICON 4th, Stellenbosch South Africa, 1996: 853-855

[3] Eftichios Koutroulis, Kostas Kalaitzakis, and Nicholas C. Voulgaris "Development of a Microcontroller-Based, Photovoltaic Maximum Power Point Tracking Control System" IEEE Transactions on Power Electrics, VOL. 16, NO. 1, Jan. 2001

[4] S. Wolf, and J. Enslin, "Economical, PV maximum power point tracking regulator with simplistic controller," IEEE Power Electron. Spec. Conf, 1993, pp. 581-587.

[5] J. Enslin, M. Wolf, D. Snyman, and W. Swiegers, "Integrated photovoltaic maximum power point tracking converter," IEEE Trans. Ind. Electron., vol. 44, no. 6, Dec. 1997, pp.769-773.

[6] Guo Heng, Xu Zheng, and Li You-Chun, "A Novel Maximum Power Point Tracking Control Method for Stand-alone Photovoltaic Pumping Systems", IEEE/PES Transmission and Distribution Conference \& Exhibition: Asia and Pacific. Dalian P. R. China, Aug. 14-18, 2005

[7] A. Al-Amoudi, and L. Zhang, "Application of radial basis function networks for solar-array modeling and maximum power-point prediction", IEE Proc. Gener. Transm. Distrib. , Vol. 147, No. 5, September 2000, pp.310-316

\section{BIOGRAPHIES}

Songbai Zhang was born in August 5th, 1979. He received B.S degree from Xi'an Jiaotong Univertiy, China in 2003. Now he studies at Department of electrical engineering, Tsinghua University, China and is engaged in research of photovoltaic applications.

Zheng Xu received B.S degree from Shanghai Jiao-tong University, China, in 1983, and M.S and PhD degrees from Kyushu Institute of Technology, Japan, in 1989 and 1993. From 1992 to 1997, he had been with the Department of Electric Engineering of Kyushu Institute of Technology as a visiting assistant professor. From 1997 to 2000, he worked at Motor Company, Matsushita Electric Industrial Co., Ltd. Since 2000, he has been with National Key Laboratory of Power Systems, Graduate School of Tsinghua University at Shenzhen, China, as an associate professor and is engaged in research on motor control and photovoltaic systems.

Youchun Li received his B.S degree from Tsinghua University in 2000. And now he is with the Shenzhen Highest Power Technology Co. Ltd.

Yixin Ni (S. M., IEEE) She received her B. Eng., M. Eng. and Ph. D. degrees all from Tsinghua University, China. She was former professor and director of National Power System Lab, Tsinghua University and Now with the University of Hong Kong. Her research interests are power system stability and control, FACTS, AI tech. applications in power systems and power market. 